Swarthmore College

Works

\title{
Improved Orbital Parameters And Transit Monitoring For HD
}

\section{$156846 b$}

S. R. Kane
A. W. Howard
G. Pilyavsky
S. Mahadevan
G. W. Henry

See next page for additional authors

Follow this and additional works at: https://works.swarthmore.edu/fac-physics

Part of the Astrophysics and Astronomy Commons

Let us know how access to these works benefits you

\section{Recommended Citation}

S. R. Kane, A. W. Howard, G. Pilyavsky, S. Mahadevan, G. W. Henry, K. Von Braun, D. R. Ciardi, D. Dragomir, D. A. Fischer, Eric L.N. Jensen, G. Laughlin, S. V. Ramirez, and J. T. Wright. (2013). "Improved Orbital Parameters And Transit Monitoring For HD 156846b". Astrophysical Journal. Volume 733, Issue 1. DOI: 10.1088/0004-637X/733/1/28

https://works.swarthmore.edu/fac-physics/46

This work is brought to you for free by Swarthmore College Libraries' Works. It has been accepted for inclusion in Physics \& Astronomy Faculty Works by an authorized administrator of Works. For more information, please contact myworks@swarthmore.edu. 


\section{Authors}

S. R. Kane, A. W. Howard, G. Pilyavsky, S. Mahadevan, G. W. Henry, K. Von Braun, D. R. Ciardi, D. Dragomir, D. A. Fischer, Eric L.N. Jensen, G. Laughlin, S. V. Ramirez, and J. T. Wright 


\title{
IMPROVED ORBITAL PARAMETERS AND TRANSIT MONITORING FOR HD 156846b
}

\author{
Stephen R. Kane ${ }^{1}$, Andrew W. Howard ${ }^{2,3}$, Genady Pilyavsky ${ }^{4}$, Suvrath Mahadevan ${ }^{4,5}$, Gregory W. Henry $^{6}$, \\ Kaspar von Braun $^{1}$, David R. Ciardi ${ }^{1}$, Diana Dragomir ${ }^{1,7}$, Debra A. Fischer ${ }^{8}$, Eric Jensen ${ }^{9}$, Gregory $^{2}$ Laughlin $^{10}$, \\ SOlange V. RAMIREZ ${ }^{1}$, AND JaSON T. WRIGHT 4,5 \\ ${ }^{1}$ NASA Exoplanet Science Institute, Caltech, MS 100-22, 770 South Wilson Avenue, Pasadena, CA 91125, USA; skane@ipac.caltech.edu \\ ${ }^{2}$ Department of Astronomy, University of California, Berkeley, CA 94720, USA \\ ${ }^{3}$ Space Sciences Laboratory, University of California, Berkeley, CA 94720, USA \\ ${ }^{4}$ Department of Astronomy and Astrophysics, Pennsylvania State University, 525 Davey Laboratory, University Park, PA 16802, USA \\ ${ }^{5}$ Center for Exoplanets \& Habitable Worlds, Pennsylvania State University, 525 Davey Laboratory, University Park, PA 16802, USA \\ ${ }^{6}$ Center of Excellence in Information Systems, Tennessee State University, 3500 John A. Merritt Blvd., Box 9501, Nashville, TN 37209, USA \\ ${ }^{7}$ Department of Physics \& Astronomy, University of British Columbia, Vancouver, BC V6T1Z1, Canada \\ ${ }^{8}$ Department of Astronomy, Yale University, New Haven, CT 06511, USA \\ ${ }^{9}$ Department of Physics \& Astronomy, Swarthmore College, Swarthmore, PA 19081, USA \\ ${ }^{10}$ UCO7/Lick Observatory, University of California, Santa Cruz, CA 95064, USA \\ Received 2011 January 23; accepted 2011 March 17; published 2011 April 29
}

\begin{abstract}
HD $156846 \mathrm{~b}$ is a Jovian planet in a highly eccentric orbit $(e=0.85)$ with a period of 359.55 days. The pericenter passage at a distance of $0.16 \mathrm{AU}$ is nearly aligned to our line of sight, offering an enhanced transit probability of $5.4 \%$ and a potentially rich probe of the dynamics of a cool planetary atmosphere impulsively heated during close approach to a bright star $(V=6.5)$. We present new radial velocity $(\mathrm{RV})$ and photometric measurements of this star as part of the Transit Ephemeris Refinement and Monitoring Survey. The RV measurements from the Keck-High Resolution Echelle Spectrometer reduce the predicted transit time uncertainty to 20 minutes, an order of magnitude improvement over the ephemeris from the discovery paper. We photometrically monitored a predicted transit window under relatively poor photometric conditions, from which our non-detection does not rule out a transiting geometry. We also present photometry that demonstrates stability at the millimagnitude level over its rotational timescale.
\end{abstract}

Key words: planetary systems - stars: individual (HD 156846) - techniques: photometric - techniques: radial velocities

Online-only material: color figure

\section{INTRODUCTION}

The discovery of exoplanets using the transit technique is becoming increasingly dominant amongst the various detection methods. Examples of major contributors to the groundbased discovery of transiting exoplanets are the Hungarian Automated Telescope Network (Bakos et al. 2004) and SuperWASP (Pollacco et al. 2006). From the vantage point of space, the major contributors are the Kepler mission (Borucki et al. 2010) and the CoRoT mission (Barge et al. 2008). The discoveries provided by these surveys are producing insights into the exoplanet mass-radius relationship, extending down toward super-Earth planets (Seager et al. 2007). Although these spacebased surveys are expected to extend the period sensitivity to longer periods, such as the case of CoRoT-9b (Deeg et al. 2010), the picture is incomplete since the surveys are strongly biased toward short-period planets around relatively faint host stars.

Several planets discovered with the radial velocity (RV) technique have subsequently been found to transit, the first of which was HD 209458b (Charbonneau et al. 2000; Henry et al. 2000). The brightness of their host stars has facilitated further characterization of their atmospheres, such as the cases of HD 189733 b and HD 149026b (e.g., Knutson et al. 2009a, 2009b; see also the review article by Seager \& Deming 2010). The Neptune-mass planet orbiting GJ 436 became the first known transiting planet around an M-dwarf primary (Gillon et al. 2007). The detection of transits for the planets HD 17156b (Barbieri et al. 2007) and HD 80606b (Laughlin et al. 2009;
Moutou et al. 2009), enabled by their high eccentricities (Kane \& von Braun 2008, 2009), provided the first insights into the structures of longer period planets. Many of the known planets with orbital periods larger than a few days have yet to be photometrically monitored at predicted transit times, hampered mostly by insufficient orbital parameter precision to accurately predict when the planet might transit. Further discoveries of long-period planetary transits around bright stars are vital to understanding the dependence of planetary structure and atmospheric dynamics on the periastron distance of the planet (Fortney et al. 2010; Kane \& Gelino 2010; Langton \& Laughlin 2008). Provided the orbital parameters can be determined with sufficient precision, monitoring planets detected via the RV technique at predicted transit times provides a means to increase the sample of long-period transiting planets (Kane et al. 2009, 2010). There exist efforts to detect transits of the known RV planets, such as the Spitzer search for transits of low-mass planets (Gillon et al. 2010). The Transit Ephemeris Refinement and Monitoring Survey (TERMS) is a program which is capable of monitoring long-period as well as short-period planets by refining the orbital parameters of the system.

Here, we present a detailed analysis of one such system. The massive planet orbiting the star HD 156846 was discovered by Tamuz et al. (2008) using the CORALIE instrument. The planet is in a highly eccentric orbit with a period of slightly less than a year. The periastron argument of the orbit is such that the transit probability is significantly enhanced compared to an equivalent circular orbit (5.4\% compared to $0.9 \%$ ). Our combined fit 
to new Keck data along with the discovery CORALIE data greatly improves the orbital parameters for the system, allowing an accurate prediction of possible transit times. We also find no evidence for additional companions in the system through high-precision RV data acquired during the periastron passage over multiple orbits. The long-term photometry presented here establishes the photometric stability of the host star. We present photometry acquired during a predicted transit window which places an upper limit on a transit for this planet. Finally, we discuss additional constraints on the mass and orbit of the planet from a potential transit null-result.

\section{SCIENCE MOTIVATION}

Here we describe why the planet orbiting HD 156846 is a particularly interesting target and the potential gains which may be achieved through further studies.

HD 156846 is an extraordinarily bright star $(V=6.5)$, brighter indeed by a factor of $\sim 2.9$ than either of the planet hosting stars HD 209458 and HD 189733. The opportunities for follow-up studies of a fundamentally new type of planetary atmosphere would therefore be close to optimal. Note that massive, relatively cold planets such as this one have intrinsically difficult atmospheres to study via transmission spectroscopy. Their atmospheric scale heights are of order a factor of 20 smaller than typical hot Jupiters (see, for example, Vidal-Madjar et al. 2011), such that a bright host star is needed to achieve adequate signal to noise.

Given the properties of this star (see Section 3.2), the received flux of the planet at apastron will be nearly identical to the flux received by the Earth from the Sun. It is therefore not unreasonable to expect that the planet during this phase of the orbit will be sheathed in reflective white water clouds. At some point prior to periastron, when the received flux increases briefly to a value nearly 150 times that at apastron, the received flux should be sufficient to flash the water clouds to steam (Sudarsky et al. 2005). Planets in the post-water cloud temperature regime are expected to have atmospheres transparent down to large pressure depths, which will cause a dramatic drop in the planet-wide albedo. Because of the extrabright primary, HD 156846 will always represent one of the very best targets in the sky for reflected light observations of this time-sensitive albedo change, which gives real insight into the atmospheric dynamics. This system is thus likely to gain in importance as photometric sensitivities improve, and so any knowledge of the inclination (and whether it transits) is very important.

Hamilton \& Burns (1992) have shown that the Hill radius at periastron is a good representation of the stability zone for a satellite of a planet in very eccentric orbit around a star. The relatively large mass of HD $156846 \mathrm{~b}$ leads to a sizable effective Hill Sphere at periastron $(\sim 0.02$ AU, $\sim 47$ Jupiter radii), indicating that detectable Earth-mass satellites orbiting the planet can be dynamically stable over the lifetime of the system if the tidal quality factor, $Q$, is of order the Jovian value or higher (Barnes \& O'Brien 2002). Given the mass ratios observed for the Jovian planets and their satellites in our own solar system, one might reasonably expect a $\sim 0.5 M_{\oplus}$ satellite, which would be readily detectable using transit timing techniques (Kipping 2009), and perhaps even directly via space-based photometry. Given the inflated transit probability for this planet, and its potentially interesting dynamical history, this becomes a prime candidate in this regard.

\section{KECK MEASUREMENTS AND REVISED ORBITAL PARAMETERS}

\subsection{Observations}

We observed HD 156846 with the High Resolution Echelle Spectrometer (HIRES; Vogt et al. 1994) on the 10 m Keck I telescope with the goal of improving the accuracy of the predicted transit time to guide and temporarily anchor a photometric monitoring campaign. Our Keck observations postdate the CORALIE measurements (Tamuz et al. 2008) and span 2009 May to 2010 October. The 41 Keck RV measurements were made from observations with an iodine cell mounted directly in front of the spectrometer entrance slit. The dense set of molecular absorption lines imprinted on the stellar spectra provides a robust wavelength fiducial against which Doppler shifts are measured, as well as strong constraints on the shape of the spectrometer instrumental profile at the time of each observation (Marcy \& Butler 1992; Valenti et al. 1995). We measured the Doppler shift of each star-times-iodine spectrum using a modeling procedure descended from Butler et al. (1996) as described in Howard et al. (2009). The times of observation (in barycentric Julian days), relative RVs, and associated errors (excluding jitter) are listed in Table 1 . In cases when we observed the star 3-5 times in quick succession, we report the mean RV and appropriately reduced uncertainty. We also observed HD 156846 with the iodine cell removed to construct a stellar template spectrum for Doppler modeling and to derive stellar properties.

\subsection{Stellar Properties}

We used Spectroscopy Made Easy (Valenti \& Piskunov 1996) to fit high-resolution Keck-HIRES spectra of HD 156846 (HIP 84856, TYC 6242-00339-1), applying the wavelength intervals, line data, and methodology of Valenti \& Fischer (2005). We further constrained the surface gravity using Yonsei-Yale $\left(\mathrm{Y}^{2}\right)$ stellar structure models (Demarque et al. 2004) and revised Hipparcos parallaxes (van Leeuwen 2007), with the iterative method of Valenti et al. (2009). The resulting stellar parameters listed in Table 2 are the effective temperature, surface gravity, iron abundance, projected rotational velocity, mass, and radius. HD 156846 lies 1.13 mag above the Hipparcos average main sequence $\left(M_{V}\right.$ versus $\left.B-V\right)$ as defined by Wright (2005). These properties are consistent with a metal-rich G0 star that evolved slightly off of the main sequence. The stellar radius, $R_{\star}=2.12 \pm 0.12 R_{\odot}$, is crucial for estimating the depth and duration of a planetary transit.

Our characterization is mostly consistent with the stellar properties reported by Tamuz et al. (2008). Although small, key differences are the larger mass $\left(M_{\star}=1.43 M_{\odot}\right)$ and higher effective temperature $\left(T_{\text {eff }}=6138 \pm 36 \mathrm{~K}\right)$ in Tamuz et al. (2008). In addition, we measured the stellar activity by measuring the strength of the $\mathrm{Ca}$ II $\mathrm{H} \& \mathrm{~K}$ lines which give calibrated $S_{\mathrm{HK}}$ values on the Mt. Wilson scale and $\log R_{\mathrm{HK}}^{\prime}$ values (Isaacson \& Fischer 2010). The medians of $\log R_{\mathrm{HK}}^{\prime}$ and $S_{\mathrm{HK}}$ values are listed in Table 2 and demonstrate that the HD 156846 is chromospherically quiet, which is consistent with the photometric stability described in Section 5.

\subsection{Keplerian Models}

With the goal of improving the orbital solution for HD $156846 \mathrm{~b}$, we constructed single-planet Keplerian models of the RVs using the orbit fitting techniques described in Howard et al. (2010) and the partially linearized, least-squares-fitting 
Table 1

Keck Radial Velocities

\begin{tabular}{|c|c|c|}
\hline BJD-2440000 & $\begin{array}{l}\text { Radial Velocity } \\
\left(\mathrm{m} \mathrm{s}^{-1}\right)\end{array}$ & $\begin{array}{c}\text { Uncertainty } \\
\left(\mathrm{m} \mathrm{s}^{-1}\right)\end{array}$ \\
\hline 14957.02010 & -111.41 & 1.94 \\
\hline 14983.95333 & -79.61 & 1.73 \\
\hline 14984.87930 & -76.60 & 1.74 \\
\hline 14985.97062 & -77.24 & 1.63 \\
\hline 14986.96751 & -78.81 & 1.69 \\
\hline 14987.91548 & -80.71 & 1.54 \\
\hline 15015.85870 & -19.24 & 1.60 \\
\hline 15016.78653 & -11.02 & 1.61 \\
\hline 15026.95355 & 22.14 & 1.29 \\
\hline 15028.96769 & 24.15 & 1.27 \\
\hline 15041.87653 & 81.78 & 1.64 \\
\hline 15042.95724 & 91.65 & 1.63 \\
\hline 15043.79662 & 94.00 & 1.56 \\
\hline 15048.77399 & 124.15 & 1.90 \\
\hline 15073.72838 & 561.70 & 1.38 \\
\hline 15074.72238 & 562.49 & 1.22 \\
\hline 15075.72238 & 507.32 & 0.72 \\
\hline 15076.73007 & 388.21 & 0.78 \\
\hline 15077.72296 & 222.03 & 0.73 \\
\hline 15078.72435 & 67.34 & 0.59 \\
\hline 15078.75583 & 61.16 & 0.57 \\
\hline 15079.72234 & -60.59 & 0.74 \\
\hline 15080.72602 & -144.09 & 0.49 \\
\hline 15081.72293 & -211.59 & 1.22 \\
\hline 15082.71457 & -252.11 & 0.86 \\
\hline 15083.71752 & -278.63 & 0.67 \\
\hline 15084.71590 & -308.49 & 1.13 \\
\hline 15111.70803 & -337.84 & 1.80 \\
\hline 15229.17709 & -213.95 & 1.67 \\
\hline 15320.98063 & -107.50 & 1.67 \\
\hline 15375.84930 & -20.06 & 1.82 \\
\hline 15402.81296 & 92.47 & 1.65 \\
\hline 15410.78620 & 144.58 & 1.51 \\
\hline 15433.71936 & 569.13 & 1.20 \\
\hline 15435.71871 & 457.38 & 0.69 \\
\hline 15439.71931 & -97.88 & 0.75 \\
\hline 15440.75164 & -174.32 & 0.70 \\
\hline 15455.70798 & -363.19 & 0.72 \\
\hline 15468.74647 & -344.65 & 1.29 \\
\hline 15471.70625 & -340.87 & 0.77 \\
\hline 15486.68538 & -316.77 & 0.92 \\
\hline
\end{tabular}

procedure described in Wright \& Howard (2009). Each velocity measurement was assigned a weight, $w$, constructed from the quadrature sum of the measurement uncertainty $\left(\sigma_{\mathrm{RV}}\right)$ and a jitter term $\left(\sigma_{\text {jitter }}\right)$, i.e., $w=1 /\left(\sigma_{\mathrm{RV}}^{2}+\sigma_{\text {jitter }}^{2}\right)$. We chose jitter values of $\sigma_{\text {jitter }}=3.38$ and $5.90 \mathrm{~m} \mathrm{~s}^{-1}$ for Keck and CORALIE to satisfy the condition $\chi_{v}^{2}=1$ for Keplerian fits to those data sets individually. These values are consistent with the expected jitter of a slightly evolved early $G$ star observed with those instruments. Sources of jitter include stellar pulsation, magnetic activity, granulation, undetected planets, and instrumental effects (Isaacson \& Fischer 2010; Wright 2005).

The Keplerian parameter uncertainties for each planet were derived using a Monte Carlo method (Marcy et al. 2005) and account for correlations between parameter errors. Specifically, our method accounts for correlations between $T_{c}$ and the other Keplerian parameters (notably $e$ ) to provide an accurate estimate of the transit time. Uncertainties in $M \sin i$ and $a$ reflect uncertainties in $M_{\star}$ and the orbital parameters.
Table 2

Stellar Properties

\begin{tabular}{lc}
\hline \hline \multicolumn{1}{c}{ Parameter } & Value \\
\hline$M_{V}$ & 3.055 \\
$B-V$ & 0.557 \\
$V$ & 6.506 \\
Distance $(\mathrm{pc})$ & $49.0 \pm 2.2$ \\
$T_{\text {eff }}(\mathrm{K})$ & $5969 \pm 44$ \\
$\log g$ & $3.92 \pm 0.08$ \\
{$[\mathrm{Fe} / \mathrm{H}]$} & $+0.17 \pm 0.04$ \\
$v \sin i\left(\mathrm{~km} \mathrm{~s}^{-1}\right)$ & $5.05 \pm 0.50$ \\
$M_{\star}\left(M_{\odot}\right)$ & $1.35 \pm 0.045$ \\
$R_{\star}\left(R_{\odot}\right)$ & $2.12 \pm 0.12$ \\
$\log R_{\mathrm{HK}}^{\prime}$ & -5.071 \\
$S_{\mathrm{HK}}$ & 0.144 \\
\hline
\end{tabular}

We considered models based on three data sets: CORALIE (Tamuz et al. 2008, 2010) and Keck-HIRES (Table 1) individually and combined. Each model consists of a single planet in Keplerian motion with the parameters listed in Table 3. We allowed for an arbitrary RV offset in the CORALIE measurements at JD 2,454,279, the time of an instrument upgrade. We also allowed for an RV offset between the Keck-HIRES and CORALIE measurements. Our models include a linear velocity trend. Because the inclusion of a trend does not lower $\chi_{v}^{2}$, the data do not provide evidence for a distant third body in the system. Nevertheless, our models include the trend to provide sufficient model flexibility to achieve properly estimated parameter uncertainties.

Table 3 lists the parameters of the three, nearly identical orbital models. Our refitting of the Tamuz et al. (2008) data has a slightly lower rms than they reported because we excluded measurements with uncertainties greater than three times the median. The higher precision Keck measurements yield a substantial improvement in the estimated parameters. The uncertainty in the predicted time of transit on 2009 September 3 is improved by an order of magnitude, from 0.227 days to 0.023 days. (This improvement is also due to the timing of the Keck measurements to coincide with that epoch.) We adopt the CORALIE + Keck model which has a period uncertainty of one part in 50,000 and a transit mid-point uncertainty of only 20 minutes.

Figure 1 shows the CORALIE and Keck measurements in time series with our adopted model overlaid. The middle panel shows Keck measurements during the 2009 September periastron passage. These measurements and similar ones during the 2010 periastron passage provide substantial leverage to constrain the orbital period and transit time. The bottom panel shows the Keck measurements on 2009 September 3, when a transit was predicted. The dotted vertical lines show the predicted midtransit time and the earliest ingress and latest egress times that are consistent with the adopted CORALIE + Keck orbital solution. These times were computed from $t_{c} \pm\left(2 \sigma_{t_{c}}+t_{\text {dur }} / 2\right)$, where $t_{c}$ and $\sigma_{t_{c}}$ are the predicted transit time and uncertainty, respectively, and $t_{\text {dur }}=490$ minutes is the duration of an equatorial transit of the star with $R_{\star}=2.12 \pm 0.12 R_{\odot}$. Our Keck measurements that night were taken as early as possible, but still missed the transit window and do not provide a constraint on the inclination from the Rossiter-McLaughlin effect (Gaudi \& Winn 2007).

\section{TRANSIT EPHEMERIS REFINEMENT}

As described by Kane \& von Braun (2008), the transit probability of a planet is a strong function of both the eccentricity 

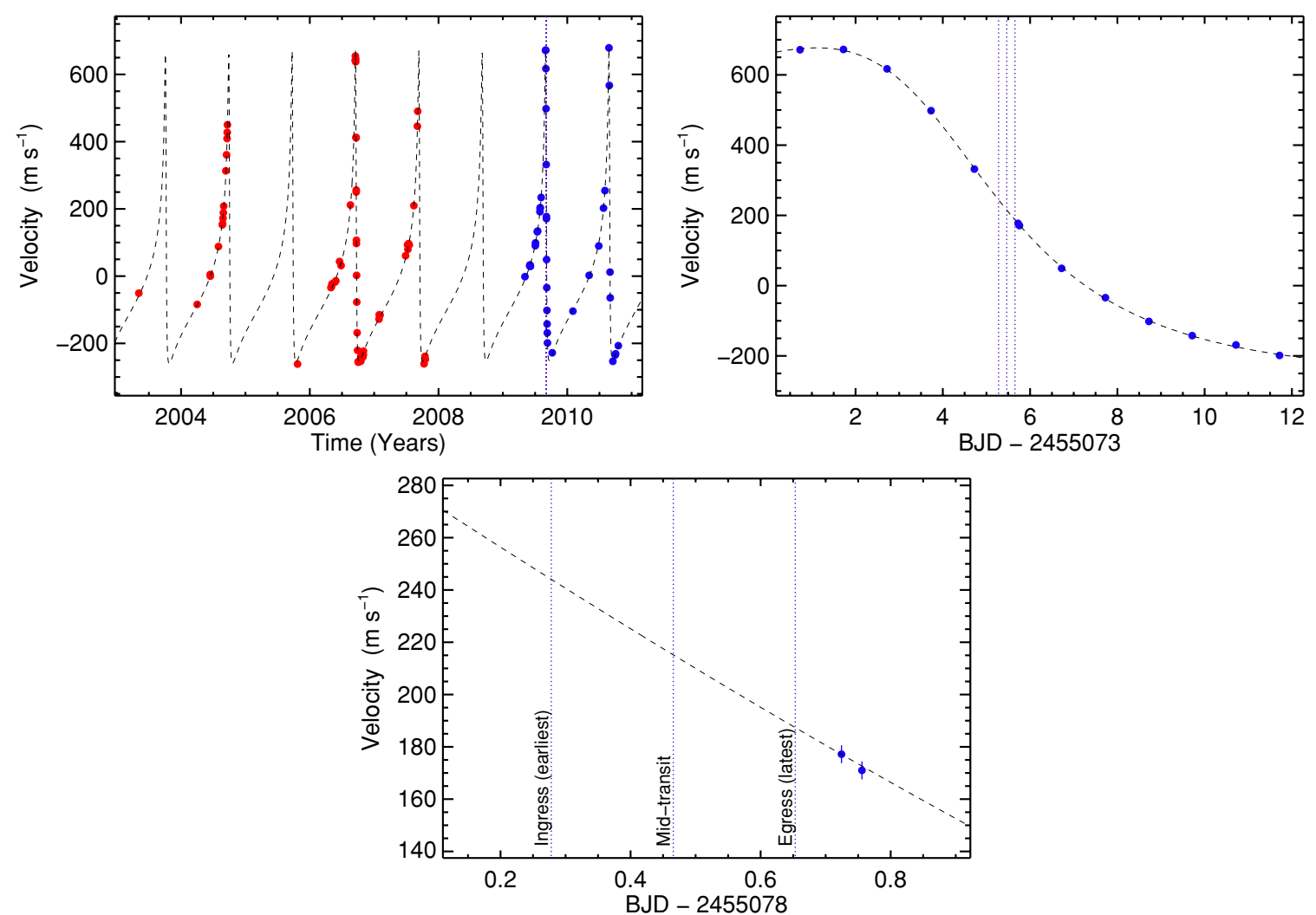

Figure 1. Time series RV measurements of HD 156846 from CORALIE (red filled circles) and Keck-HIRES (blue filled circles). The adopted Keplerian orbital solution (Table 3) is shown as a dashed line in all panels. Dotted vertical lines illustrate the times of ingress, mid-transit, and egress for a predicted time of transit on 2009 September 3 (see the text). Top: all CORALIE and Keck-HIRES measurements. Middle: Keck measurements during the 2009 September periastron passage. Bottom: Keck measurements on 2009 September 3.

(A color version of this figure is available in the online journal.)

Table 3

Keplerian Orbital Models

\begin{tabular}{lccc}
\hline \hline \multicolumn{1}{c}{ Parameter } & CORALIE & Keck & CORALIE+ Keck (adopted) \\
\hline$P($ days $)$ & $359.5400 \pm 0.1072$ & $359.5517 \pm 0.0270$ & $359.5546 \pm 0.0071$ \\
$T_{c}{ }^{a}(\mathrm{JD}-2,440,000)$ & $15078.459 \pm 0.219$ & $15078.470 \pm 0.017$ & $15078.483 \pm 0.015$ \\
$T_{p}{ }^{b}(\mathrm{JD}-2,440,000)$ & $15076.699 \pm 0.227$ & $15076.671 \pm 0.023$ & $15076.686 \pm 0.021$ \\
$e$ & $0.84696 \pm 0.00109$ & $0.84828 \pm 0.00061$ & $0.84785 \pm 0.00050$ \\
$K\left(\mathrm{~m} \mathrm{~s}^{-1}\right)$ & $464.03 \pm 2.14$ & $463.64 \pm 1.20$ & $464.14 \pm 0.96$ \\
$\omega(\mathrm{deg})$ & $52.51 \pm 0.39$ & $51.42 \pm 0.18$ & $51.62 \pm 0.16$ \\
$d v / d t\left(\mathrm{~m} \mathrm{~s}^{-1} \mathrm{yr}^{-1}\right)$ & $3.51 \pm 1.41$ & $0.86 \pm 1.36$ & $1.55 \pm 0.88$ \\
$M \sin i\left(M_{\mathrm{Jup}}\right)$ & $10.60 \pm 0.29$ & $10.54 \pm 0.29$ & $10.57 \pm 0.29$ \\
$a(\mathrm{AU})$ & $1.096 \pm 0.021$ & $1.096 \pm 0.021$ & $1.096 \pm 0.021$ \\
$\mathrm{rms}\left(\mathrm{m} \mathrm{s}^{-1}\right)$ & 6.85 & 3.45 & 6.06
\end{tabular}

Notes.

a Time of transit.

b Time of periastron passage.

and the argument of periastron. In particular, the transit probability is the strongest when the periastron passage occurs close to the star-observer line of sight, or where $\omega=90^{\circ}$. The orbit of HD 156846b, shown in Figure 2, is well suited for photometric follow-up at predicted transit time since the alignment of the major axis with the observer clearly leads to an enhanced transit probability.

From the derived stellar properties shown in Table 2 and the planetary properties from Table 3 , we derive a planetary radius of $R_{p}=1.1 R_{J}$ using the methods described in Bodenheimer et al. (2003). This produces a transit probability of $5.4 \%$ and a predicted transit depth of 3 mmag. The uncertainty in the stellar mass/radius and subsequent uncertainty in the planetary mass/ radius have a minor effect on the estimated transit duration but in no way affects the predicted transit mid-points since these are derived from the orbital parameters (Kane et al. 2009). Based upon the revised orbital parameters, the transit ephemeris has been calculated for the period 2009-2016 and is shown in Table 4.

As described in the previous section, the size of the transit window for 2009 was 0.37 days, or $\sim 9 \mathrm{hr}$. The uncertainty on the transit mid-point is small, $\sim 20$ minutes, so the transit window is largely dominated by the transit duration. The small uncertainty on the period ensures that the size of the transit window does not 
Table 4

Refined Transit Ephemeris for HD 156846b

\begin{tabular}{|c|c|c|c|c|c|}
\hline \multicolumn{2}{|c|}{ Beginning } & \multicolumn{2}{|c|}{ Mid-point } & \multicolumn{2}{|c|}{ End } \\
\hline JD & Date & JD & Date & JD & Date \\
\hline 2455078.30 & 200909031909 & 2455078.48 & 200909032335 & 2455078.67 & 200909040401 \\
\hline 2455437.85 & 201008290817 & 2455438.04 & 201008291254 & 2455438.23 & 201008291730 \\
\hline 2455797.39 & 201108232125 & 2455797.59 & 201108240212 & 2455797.79 & 201108240659 \\
\hline 2456156.94 & 201208171034 & 2456157.15 & 201208171531 & 2456157.35 & 201208172028 \\
\hline 2456516.49 & 201308112342 & 2456516.70 & 201308120449 & 2456516.91 & 201308120957 \\
\hline 2456876.04 & 201408061250 & 2456876.26 & 201408061808 & 2456876.48 & 201408062326 \\
\hline 2457235.58 & 201508010159 & 2457235.81 & 201508010727 & 2457236.04 & 201508011255 \\
\hline 2457595.13 & 201607251507 & 2457595.37 & 201607252045 & 2457595.60 & 201607260223 \\
\hline
\end{tabular}

Notes. The columns indicate the beginning, mid-point, and end of the transit window in both Julian and calendar date. The calendar date is expressed in UT and includes the year, month, day, hour, and minute.

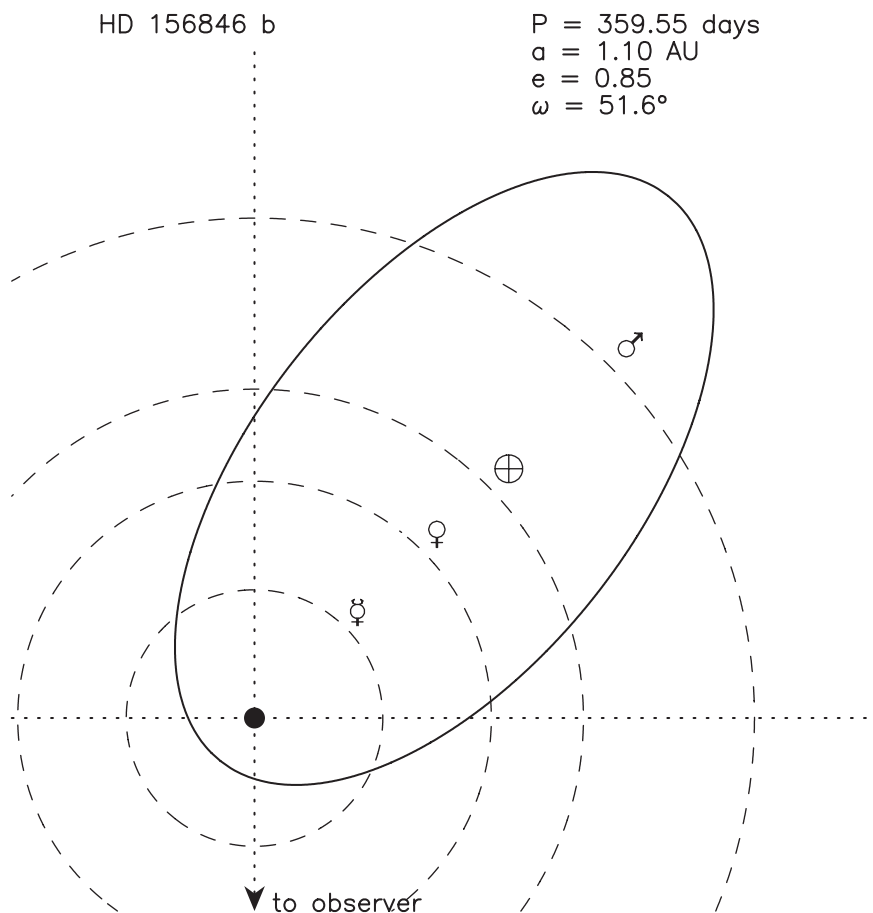

Figure 2. Orbit of the planet orbiting HD 156846 (solid line) and the orbits of the solar system planets for comparison (dashed lines).

grow substantially with time. In 2016, for example, the transit window is only $2.5 \mathrm{hr}$ longer than it was in 2009. The transit duration makes it very difficult to attempt complete coverage of the transit window without a multi-longitudinal campaign and exceptional weather conditions. However, ground-based observations of either ingress or egress will be feasible from a given observing location, provided photometric precision requirements are met.

\section{PHOTOMETRIC STABILITY}

Here, we describe photometry acquired outside of the transit window for the purposes of studying the stability of the star. The only source of time-series photometry for HD 156846 in the literature is the Hipparcos catalog (Perryman et al. 1997). HD 156846 (HIP 84856) was measured 82 times over the three-year duration of the Hipparcos mission; its variability classification in the catalog is blank, indicating that the star "could not be classified as variable or constant." The scatter (standard deviation) of the 82 observations was 0.007 mag.
We acquired new photometry of HD 156846 with the T8 0.80 $\mathrm{m}$ automatic photometric telescope (APT) at Fairborn Observatory in southern Arizona (Henry 1999). T8 uses a two-channel precision photometer with two EMI 9124QB bi-alkali photomultiplier tubes to make simultaneous measurements in the Strömgren $b$ and $y$ passbands. The telescope was programmed to make differential brightness measurements of the program star P (HD 156846, $V=6.50, B-V=0.58, \mathrm{G} 1 \mathrm{~V}$ ) with respect to the two comparison stars $\mathrm{C} 1$ (HD 157379, $V=6.65$, $B-V=0.43, \mathrm{~F} 3 \mathrm{IV}-\mathrm{V}$ ) and C2 (HD 156058, $V=7.70$, $B-V=0.48, \mathrm{~F} 3 \mathrm{~V})$. To improve the precision of our differential magnitudes, we averaged the $b$ and $y$ observations to create a $(b+y) / 2$ "passband." The typical precision of a single observation in this combined passband is $0.0015 \mathrm{mag}$, as measured from pairs of constant stars (Henry 1999).

Between 2010 June 1 and July 6, the APT collected 32 good measurements of the $\mathrm{P}-\mathrm{C} 1, \mathrm{P}-\mathrm{C} 2$, and $\mathrm{C} 2-\mathrm{C} 1$ differential magnitudes with standard deviations of $0.00219,0.00229$, and $0.00202 \mathrm{mag}$, respectively, slightly higher than the typical 0.0015 mag precision. However, HD 156846 and its comparison stars are located between $-16^{\circ}$ and $-19^{\circ}$ decl., so they are observed at higher than average air mass. The observed scatter of the $\mathrm{P}-\mathrm{C} 1, \mathrm{P}-\mathrm{C} 2$, and $\mathrm{C} 2-\mathrm{C} 1$ differential magnitudes are all consistent with constant stars. Periodogram analyses of the three data sets reveal no significant periodicity. We conclude that HD 156846 is constant on its rotation timescale.

\section{MONITORING THE TRANSIT WINDOW}

HD 156846 was observed at the Cerro Tololo Inter-American Observatory $1.0 \mathrm{~m}$ telescope using the Y4KCam Detector, which is a $4 \mathrm{k} \times 4 \mathrm{k}$ CCD with a field of view of about 20 arcmin on the side. ${ }^{11}$ We monitored HD 156846 in the Johnson $B$ band on the nights of 2009 September 3-during its transit-and on September 4, 8, 9, 11, and 12 for out-oftransit calibration purposes. Due to HD 156846's brightness ( $V=6.506 ; B=7.063$ ), we used a diaphragm in the shape of a ring constructed of plywood to decrease the effective aperture of the telescope, thereby reducing flux by around $40 \%$, similar to the technique employed by López-Morales (2006). For the observing strategy and photometry, our methods are similar to those described by Southworth et al. (2009). To ensure that no part of the stellar point-spread function (PSF) reaches the nonlinearity levels of the CCD, and to maximize the number of counts contained in the PSF, we defocused the telescope such that the full-width at half-maximum of the PSF subtended 3-8

\footnotetext{
11 http://www.astronomy.ohio-state.edu/Y4KCam/
} 


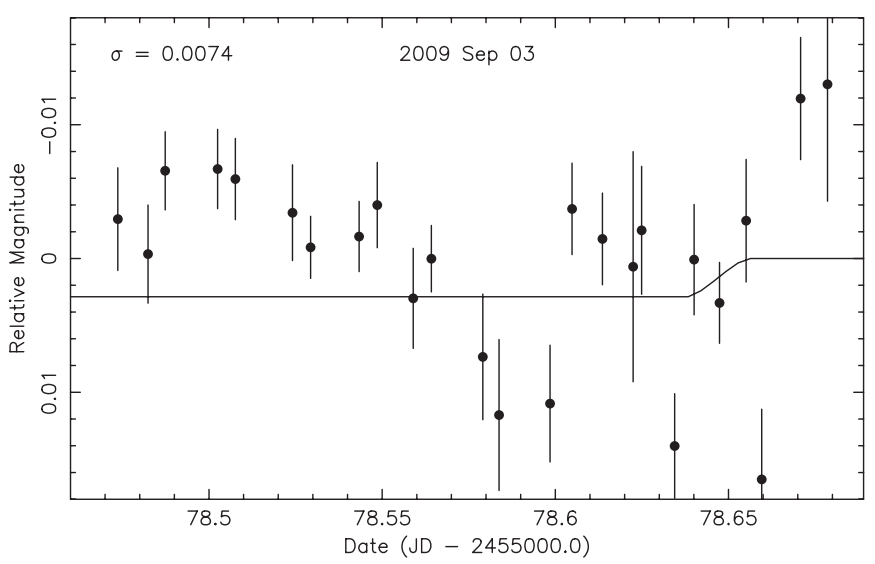

Figure 3. Binned photometry of HD 156846 from the night of the transit window. The overlaid solid line shows the predicted transit signature.

arcsec, which produced $\sim 10^{6}$ ADUs (and thus 1 mmag photon noise) per PSF per measurement. Observing conditions on the night of the transit window (2009 September 3) were not optimal and plagued with thin cirrus. We selected the brightest four reference stars in the frame and relative photometry was performed using the methods described in Everett \& Howell (2001).

The new calculated stellar radius presented in this paper is $2.12 R_{\odot}$, significantly larger than predictions based purely upon the spectral type and luminosity class of the star. This has three primary effects on the transit prediction. The first is to increase the transit probability to $5.4 \%$. The second is to decrease the predicted transit depth to $3 \mathrm{mmag}$. The third is to increase the predicted transit duration to $\sim 9 \mathrm{hr}$. The last two aspects are particularly harmful to attempts at detecting a transit since they increase the photometric precision requirements and decrease the chances that the window can be monitored while the target is observable.

Figure 3 shows the photometry from the night of the transit window. To improve the rms scatter, we binned the data into 30 equal time intervals. This improved the $1 \sigma$ scatter from $9 \mathrm{mmag}$ to $7 \mathrm{mmag}$. Unfortunately, the poor conditions on that night prevented the necessary precision from being achieved since this is still a factor of two greater than the predicted transit depth. We calculated the predicted transit signature based upon the analytic models of Mandel \& Agol (2002), overplotted as a solid line in the figure. For a more detailed study on the effect of eccentric orbits on transit light curves, we refer the reader to Kipping $(2008,2010)$. Although we see no evidence for a transit in our data, the photometric precision is inadequate to rule out such an event. For the stellar radius we adopt, the predicted transit depth is likely to be quite robust against variations in the planetary radius. Fortney et al. (2007) showed that, for a given planetary composition, planetary radii should not vary substantially between orbital radii of 0.1 and 2.0 AU. In order to produce a transit depth comparable to the level of precision on the night of the transit window, the radius of the companion would need to be $>1.8$ Jupiter radii. Furthermore, the increase in the estimated stellar radius caused the predicted end of the transit to occur when the light curve is heavily influenced by both high air mass and cloud contamination. Thus, improved precision would not have allowed sufficient in and out of transit data to be acquired in order to comfortably secure the detection.

If a transit of this planet were to be ruled out, then weak constraints on the inclination of the orbit could be placed.

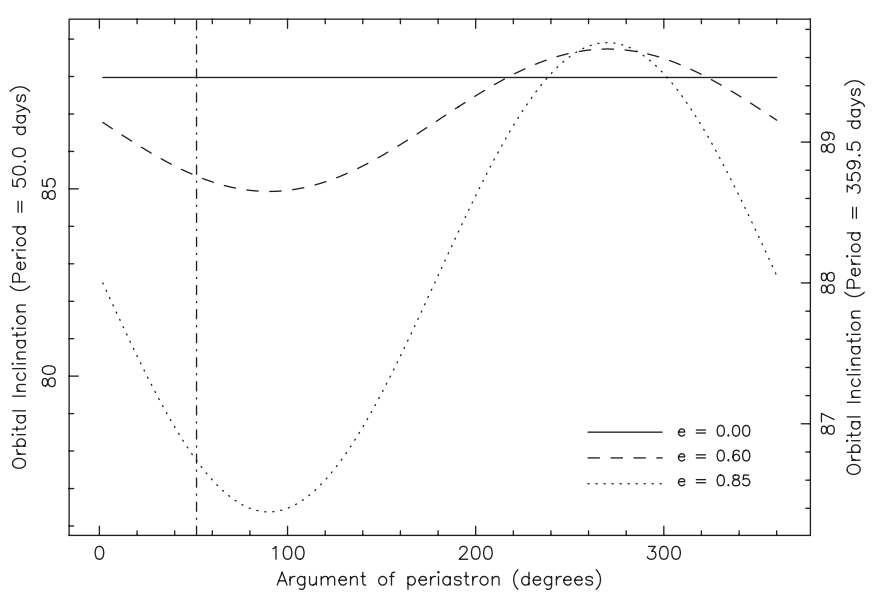

Figure 4. Maximum orbital inclination for a non-transiting planet as a function of the argument of periastron for eccentricities of 0.0 (solid line), 0.6 (dashed line), and 0.85 (dotted line), plotted for periods of both 50.0 days and 359.5 days. The vertical line indicates the location of the measured periastron argument reported here.

The magnitude of these constraints as a function of periastron argument is shown in Figure 4 which shows the maximum orbital inclination for two different periods and three different eccentricities, including the period and eccentricity of HD $156846 \mathrm{~b}$. A successful null-detection would limit the inclination to $i<86.5$ which would thus place a lower limit on the planetary mass of 10.59 Jupiter masses. The period of 50 days is shown for comparative purposes, where one can rapidly improve the lower mass limits for the smaller star-planet separation.

\section{CONCLUSIONS}

This study has been carried out as part of the TERMS. The purpose of the presented research was to improve the orbital parameters of the known exoplanet HD 156846b and to monitor the transit window. We present new Keck data which, combined with previously acquired CORALIE data, refine the orbital parameters of the planet. The measurements obtained during the periastron passage have allowed us to construct an exceptionally accurate transit ephemeris which we present here up until the year 2016. The value in a successful transit detection would be high for such a long-period planet in an eccentric orbit since it would provide insight into the mass-radius relationship for planets in this regime as well as allow follow-up characterization to determine the radiative timescale and other properties of the atmosphere.

The challenge of attempting to monitor the transit window is substantial since predicted transit windows are so infrequent, the predicted transit duration is relatively long, and photometry of bright stars becomes complicated when comparison stars are few. We present one such attempt here where observations were undertaken during non-photometric conditions which does not allow the transit to be decisively ruled out. A more suitable facility to use for such a search is the Microvariability and Oscillations of Stars satellite, such as that carried out by Croll et al. 2007. From the ground, the planned telescopes and instruments of the Las Cumbres Observatory Global Telescope (LCOGT) Network (Shporer et al. 2010) will be ideal for transit monitoring due to both their aperture size and longitude coverage. The recent astrometry work of Reffert \& Quirrenbach (2011) appears to indicate that HD 156846 b may not be in an edge-on orbit, but the results are uncertain enough to make 
this study a worthwhile exercise. We thus encourage future observations of transit windows for this planet for those cases where the window is aligned with the visibility of the target. Since the period of the planet is slightly less than one year, this situation will gradually improve with each successive transit window.

The authors thank the anonymous referee, whose comments greatly improved the quality of the paper. This work made use of the SIMBAD database (operated at CDS, Strasbourg, France), NASA's Astrophysics Data System Bibliographic Services, and the NASA Star and Exoplanet Database (NStED). This work was partially supported by funding from the Center for Exoplanets and Habitable Worlds. The Center for Exoplanets and Habitable Worlds is supported by the Pennsylvania State University, the Eberly College of Science, and the Pennsylvania Space Grant Consortium. Finally, the authors wish to extend special thanks to those of Hawai'ian ancestry on whose sacred mountain of Mauna Kea we are privileged to be guests. Without their generous hospitality, the Keck observations presented herein would not have been possible.

\section{REFERENCES}

Bakos, G., Noyes, R. W., Kovács, G., Stanek, K. Z., Sasselov, D. D., \& Domsa, I. 2004, PASP, 116, 266

Barbieri, M., et al. 2007, A\&A, 476, L13

Barge, P., et al. 2008, A\&A, 482, L17

Barnes, J. W., \& O'Brien, D. P. 2002, ApJ, 575, 1087

Bodenheimer, P., Laughlin, G., \& Lin, D. N. C. 2003, ApJ, 592, 555

Borucki, W. J., et al. 2010, Science, 327, 977

Butler, R. P., Marcy, G. W., Williams, E., McCarthy, C., Dosanjh, P., \& Vogt, S. S. 1996, PASP, 108, 500

Charbonneau, D., Brown, T. M., Latham, D. W., \& Mayor, M. 2000, ApJ, 529, L45

Croll, B., et al. 2007, ApJ, 658, 1328

Deeg, H. J., et al. 2010, Nature, 464, 384

Demarque, P., Woo., J., Kim, Y., \& Yi, S. K. 2004, ApJS, 155, 667

Everett, M. E., \& Howell, S. B. 2001, PASP, 113, 1428

Fortney, J. J., Marley, M. S., \& Barnes, J. W. 2007, ApJ, 659, 1661

Fortney, J. J., Shabram, M., Showman, A. P., Lian, Y., Freedman, R. S., Marley,

M. S., \& Lewis, N. K. 2010, ApJ, 709, 1396

Gaudi, B. S., \& Winn, J. N. 2007, ApJ, 655, 550

Gillon, M., et al. 2007, A\&A, 472, L13

Gillon, M., et al. 2010, A\&A, 518, 25

Hamilton, D. P., \& Burns, J. A. 1992, Icarus, 96, 43
Henry, G. W. 1999, PASP, 111, 845

Henry, G. W., Marcy, G. W., Butler, R. P., \& Vogt, S. S. 2000, ApJ, 529, L41

Howard, A. W., et al. 2009, ApJ, 696, 75

Howard, A. W., et al. 2010, ApJ, 721, 1467

Isaacson, H., \& Fischer, D. A. 2010, ApJ, 725, 875

Kane, S. R., \& Gelino, D. M. 2010, ApJ, 724, 818

Kane, S. R., Mahadevan, S., von Braun, K., Laughlin, G., \& Ciardi, D. R. 2009, PASP, 121, 1386

Kane, S. R., Reffert, S., Henry, G. W., Fischer, D., Schwab, C., Clubb, K. I., \& Bergmann, C. 2010, ApJ, 720, 1644

Kane, S. R., \& von Braun, K. 2008, ApJ, 689, 492

Kane, S. R., \& von Braun, K. 2009, PASP, 121, 1096

Kipping, D. M. 2008, MNRAS, 389, 1383

Kipping, D. M. 2009, MNRAS, 392, 181

Kipping, D. M. 2010, MNRAS, 407, 301

Knutson, H. A., Charbonneau, D., Cowan, N. B., Fortney, J. J., Showman, A. P., Agol, E., \& Henry, G. W. 2009a, ApJ, 703, 769

Knutson, H. A., et al. 2009b, ApJ, 690, 822

Langton, J., \& Laughlin, G. 2008, ApJ, 674, 1106

Laughlin, G., Deming, D., Langton, J., Kasen, D., Vogt, S., Butler, P., Rivera, E., \& Meschiari, S. 2009, Nature, 457, 562

López-Morales, M. 2006, PASP, 118, 716

Mandel, K., \& Agol, E. 2002, ApJ, 580, L171

Marcy, G. W., \& Butler, R. P. 1992, PASP, 104, 270

Marcy, G. W., Butler, R. P., Vogt, S. S., Fischer, D. A., Henry, G. W., Laughlin, G., Wright, J. T., \& Johnson, J. A. 2005, ApJ, 619, 570

Moutou, et al. 2009, A\&A, 498, L5

Perryman, M. A. C., et al. 1997, The Hipparcos and Tycho Catalogues (ESA SP-1200; Noordwijk: ESA)

Pollacco, D. L., et al. 2006, PASP, 118, 1407

Reffert, S., \& Quirrenbach, A. 2011, A\&A, 527, 140

Seager, S., \& Deming, D. 2010, ARA\&A, 48, 631

Seager, S., Kuchner, M., Hier-Majumder, C. A., \& Militzer, B. 2007, ApJ, 669, 1279

Shporer, A., Brown, T., Lister, T., Street, R., Tsapras, Y., Bianco, F., Fulton, B., \& Howell, A. 2010, in IAU Symp. 276, The Astrophysics of Planetary Systems: Formation, Structure, and Dynamical Evolution, ed. A. Sozzetti, M. G. Lattanzi, \& A. P. Boss (Cambridge: Cambridge Univ. Press), in press Southworth, J., et al. 2009, MNRAS, 396, 1023

Sudarsky, D., Burrows, A., Hubeny, I., \& Li, A. 2005, ApJ, 627, 520

Tamuz, O., et al. 2008, A\&A, 480, L33

Tamuz, O., et al. 2010, VizieR Online Data Catalog, 348, 9033

Valenti, J. A., Butler, R. P., \& Marcy, G. W. 1995, PASP, 107, 966

Valenti, J. A., \& Fischer, D. A. 2005, ApJS, 159, 141

Valenti, J. A., \& Piskunov, N. 1996, A\&AS, 118, 595

Valenti, J. A., et al. 2009, ApJ, 702, 989

van Leeuwen, F. 2007, A\&A, 474, 653

Vidal-Madjar, A., et al. 2011, A\&A, 527, 110

Vogt, S. S., et al. 1994, Proc. SPIE, 2198, 362

Wright, J. T. 2005, PASP, 117, 657

Wright, J. T., \& Howard, A. W. 2009, ApJS, 182, 205 